\title{
Microscopic description of quadrupole-octupole coupling in actinides with the Gogny-D1M energy density functional
}

\author{
R. Rodríguez-Guzmán' and Y. M. Humadi \\ Department of Physics, Kuwait University, Kuwait \\ L.M. Robledo \\ Center for Computational Simulation, Universidad Politécnica de Madrid, \\ Campus Montegancedo, 28660 Boadilla del Monte, Madrid, Spain and \\ Departamento de Fúsica Teórica and CIAFF, Universidad Autónoma de Madrid, 28049-Madrid, Spain
}

(Dated: June 15, 2021)

\begin{abstract}
The interplay between quadrupole and octupole degrees of freedom is discussed in a series of U, $\mathrm{Pu}, \mathrm{Cm}$ and $\mathrm{Cf}$ isotopes both at the mean-field level and beyond. In addition to the static HartreeFock-Bogoliubov approach, dynamical beyond-mean-field correlations are taken into account via both parity restoration and symmetry-conserving Generator Coordinate Method calculations based on the parametrization D1M of the Gogny energy density functional. Physical properties such as correlation energies, negative-parity excitation energies as well as reduced transition probabilities $B(E 1)$ and $B(E 3)$ are discussed in detail and compared with the available experimental data. It is shown that, for the studied nuclei, the quadrupole-octupole coupling is weak and to a large extent the properties of negative parity states can be reasonably well described in terms of the octupole degree of freedom alone.
\end{abstract}

PACS numbers: 21.60.Jz, 27.70.+q, 27.80.+w

\section{INTRODUCTION.}

Fingerprints of octupole collectivity in even-even nuclei are usually associated with the presence of $1^{-}$states in the low-lying spectra. As the ground state of those nuclei is usually quadrupole deformed, there is a $3^{-}$state, member of the corresponding negative-parity rotational bands, which decay through fast E3 transitions to the $0^{+}$ground state. On the other hand, the $1^{-}$state decays via $E 1$ transitions. The exploration of these as well as other unusual features associated with octupole correlations [1 3] already started in the 1980s [4] and has become an active field of research since then - see Refs [5] for some recent examples. In 6] the measured $E 3$ strength in ${ }^{220} \mathrm{Rn}$ and ${ }^{224} \mathrm{Ra}$ unambiguously established the octupole deformed character of the later nucleus. This represents the first unambiguous experimental evidence of permanent octupole deformed even-even nucleus. In multi-step Coulomb excitation experiments performed at the ATLAS-CARIBU facility with $\gamma$-ray and charged-particle detectors 9 also large $E 3$ transition strength in ${ }^{144} \mathrm{Ba}$ was found pointing to a permanent octupole deformed ground-state. Evidence for permanent octupole deformation in ${ }^{146} \mathrm{Ba}$ has subsequently been obtained [10. Recent experiments [11] have also established the octupole deformed character of ${ }^{222} \mathrm{Ra}$, or measured the $E 1$ strength in ${ }^{228} \mathrm{Th}[12$.

From a theoretical point of view, various techniques and models have been employed to study the dynamics of

\footnotetext{
*Electronic address: raynerrobertorodriguez@gmail.com
}

${ }^{\dagger}$ Electronic address: luis.robledo@uam.es octupole collectivity [4, 13 18. Some of the approaches use potential energy surfaces (PESs) obtained within relativistic and nonrelativistic mean-field approximations to obtain the parameters of the Interacting Boson Model 1922 . Some others rely on microscopic frameworks, both at the mean-field level and beyond, based on the nonrelativistic Skyrme and Gogny as well as relativistic energy density functionals (EDFs) 23 43.

Octupole deformation properties of several even-even actinides were discussed in Ref. [44 with the help of octupole-constrained Hartree-Fock-Bogoliubov (HFB) calculations based on the parametrizations D1S [45, D1N [46 and D1M 47] of the Gogny 48 and the BCP 49] 52 EDFs. A one-dimensional (1D) collective Hamiltonian was also built to have access to properties such as the excitation energies of $1^{-}$states as well as $B(E 1)$ and $B(E 3)$ transition probabilities. A thorough account over a large set of even-even nuclei of observables associated to octupole correlations was presented in Refs. [53, 54 using the octupole-constrained Gogny-HFB approach, parity projection and octupole configuration mixing. From the results of these studies it is clear that not only static octupole deformation plays a role but also dynamical octupole correlations have a sizable impact on observables.

The interplay between quadrupole transitional properties and octupole deformation manifestations in a selected set of Sm and Gd nuclei was discussed in Ref. [55] using the D1S and D1M Gogny-EDFs. Both quadrupole and octupole constrains were considered simultaneously. The mean-field potential energy surfaces (MFPESs) obtained for ${ }^{146-154} \mathrm{Sm}$ and ${ }^{148-156} \mathrm{Gd}$ exhibited a very soft behavior along the octupole direction indicating, that dynamical beyond-mean-field effects should be taken into account. Those beyond-mean-field effects were consid- 

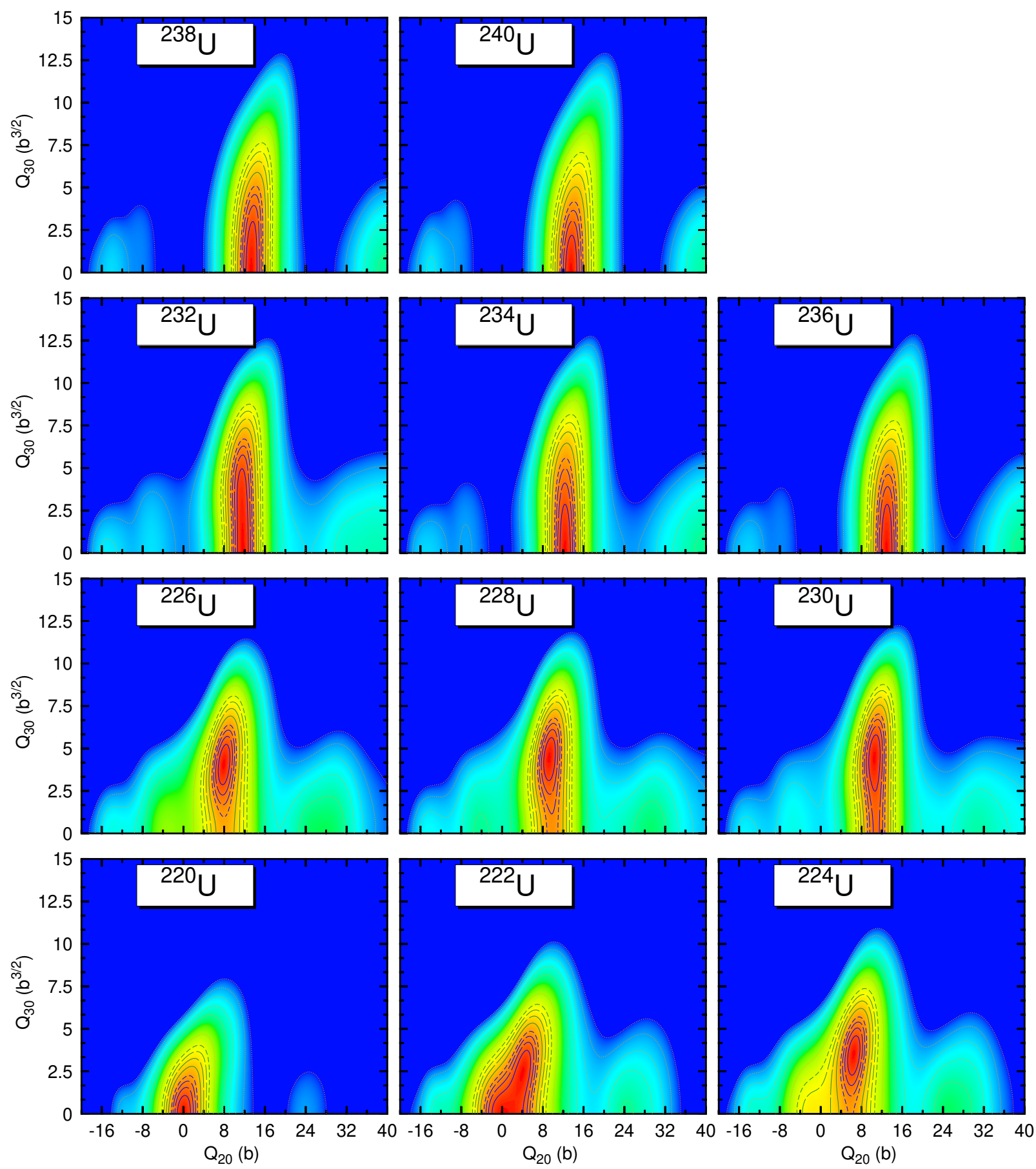

FIG. 1: (Color online) MFPESs computed with the Gogny-D1M EDF for the isotopes ${ }^{220-240}$ U. Taking the lowest mean-field energy as a reference, solid and dashed contour lines extend from $0.25 \mathrm{MeV}$ up to $1 \mathrm{MeV}$ in steps of $0.25 \mathrm{MeV}$. Solid and dashed contours are then drawn in steps of $0.5 \mathrm{MeV}$ up to $3 \mathrm{MeV}$ and from there up dotted lines are drawn in steps of $1 \mathrm{MeV}$. The intrinsic HFB energies are symmetric under the exchange $Q_{30} \rightarrow-Q_{30}$. For $A=230$, the conversion factor from barn to $\beta_{2}$ values is 0.0212 and the one from $b^{3 / 2}$ to $\beta_{3}$ values is 0.0342 . For additional details, see the main text.

ered via both parity projection of the intrinsic states and symmetry-conserving quadrupole-octupole configuration mixing calculations, in the spirit of the two-dimensional (2D) Generator Coordinate Method (GCM) [56. In addition to the systematic of the $1^{-}$excitation energies, correlation energies, $B(E 1)$ and $B(E 3)$ transition probabili- ties, the results of Ref. [55] suggested a shape/phase transition from weakly to well quadrupole deformed ground states as well as a transition to an octupole vibrational regime in the studied nuclei. The quadrupole-octupole coupling has also been studied for Rn, Ra and Th nuclei within the 2D-GCM framework [57]. Let us also mention 
a recent state-of-the-art quadrupole-octupole symmetryprojected configuration mixing study for ${ }^{144} \mathrm{Ba}$ [43].

Given the experimental interest in studying octupole properties of nuclei heavier than $\mathrm{Th}$, we consider in the present work the dynamical interplay between quadrupole and octupole degrees of freedom in a selected set of even-even actinides, i.e., ${ }^{220-240} \mathrm{U},{ }^{222-242} \mathrm{Pu}$, ${ }^{222-242} \mathrm{Cm}$ and ${ }^{222-242} \mathrm{Cf}$. These nuclei have $Z$ values away from $Z=88$ ( $\mathrm{Ra}$ ) which is considered to be a "magic number" for the existence of permanent octupole deformation [4]. The study of the dynamical quadrupoleoctupole coupling in the selected actinide nuclei allows us to examine the role of the corresponding zero-point quantum fluctuations on the systematic of the $1^{-}$excitation energies, transition strengths and correlation energies around the $N=134$ (a neutron octupole magic number) isotones ${ }^{226} \mathrm{U},{ }^{228} \mathrm{Pu},{ }^{230} \mathrm{Cm}$ and ${ }^{232} \mathrm{Cf}$.

As in our previous study [55], we consider three levels of approximation for each of the studied nuclei. The constrained Gogny-HFB scheme is used to obtain MFPESs as functions of both the quadrupole and octupole moments. As discussed later, those MFPESs can be rather soft along the octupole direction. Some of the considered nuclei also exhibit transitional features along the quadrupole direction. In this case the HFB approximation can only be considered as a starting point and beyond-mean-field correlations should be taken into account. First, parity projection is carried out in order to build the corresponding parity-projected potential energy surfaces (PPPESs). Next, both symmetry restoration as well as fluctuations in the collective quadrupole and octupole coordinates are taken into account within the 2D-GCM framework. Although reflection symmetry is also restored by our GCM ansatz (see, Sec. IIC), the parity-projected results allow us to disentangle the relative contribution to the total correlation that has to be associated with the restoration of the reflection symmetry.

All the results discussed in this paper have been obtained with the Gogny-D1M EDF [47. Among the members of the D1 family of parametrizations of the Gogny-EDF, D1S [45] has already built a strong reputation among practitioners, given its ability to reproduce a wealth of low-energy nuclear data all over the nuclear chart both at the mean-field level and beyond (see, for example, Ref. [58] and references therein). Nevertheless, the parametrization D1M, specially tailored to better describe nuclear masses, has already provided a reasonable description of nuclear properties in different regions of the nuclear chart (see, for example, Refs. 59 62 and references therein). In particular, previous studies [44, 53, 55, 57] have shown that the parametrization D1M essentially keeps the same predictive power as D1S when applied to the description of octupole properties.

The paper is organized as follows. The different approaches employed in this work are briefly outlined in Secs. II A, IIB and II C. In each section the results obtained with the corresponding approaches are discussed.

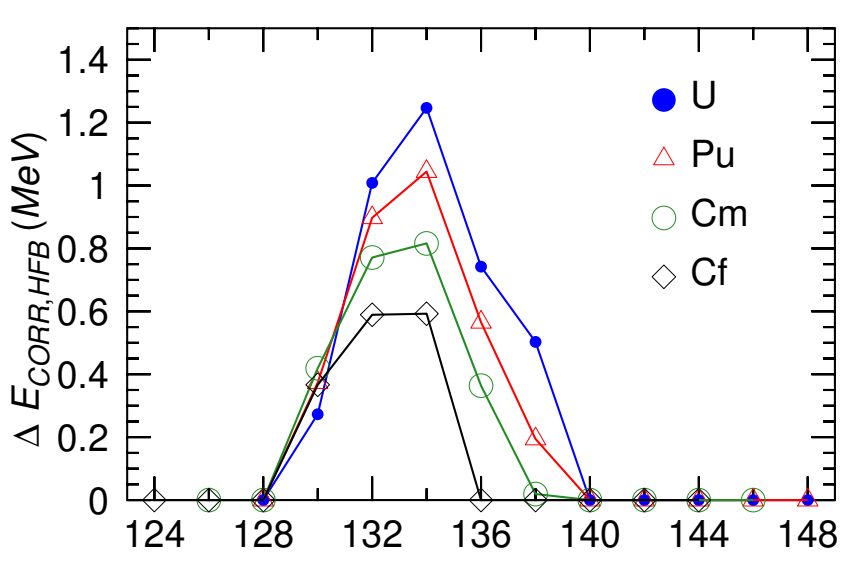

FIG. 2: (Color online) The mean-field octupole correlation energies Eq. (3) are plotted as functions of the neutron number. Results have been obtained with the Gogny-D1M EDF. For more details, see the main text.

Mean-field results are presented in Sec. II A. We then turn our attention to beyond-mean-field properties, i.e., parity restoration and configuration mixing in Secs. II B and II C. Special attention is paid in Sec. II C to $1^{-}$energy splittings, reduced transition probabilities, correlation energies and their comparison with the available experimental data 63. Finally, Sec. III is devoted to the concluding remarks.

\section{RESULTS}

The aim of this work is to study the quadrupoleoctupole dynamics in a selected set of actinide nuclei. Three levels of approximation have been considered: the HFB approach [56] with constrains on the (axially symmetric) quadrupole and octupole operators, parity projection and the 2D-GCM. In what follows, we outline those approaches [55, 57, based on the Gogny-D1M EDF, and discuss the results obtained with each of them.

\section{A. Mean-field}

To obtain the MFPESs, the HFB equation with constrains on the axially symmetric quadrupole

$$
\hat{Q}_{20}=z^{2}-\frac{1}{2}\left(x^{2}+y^{2}\right)
$$

and octupole operator

$$
\hat{Q}_{30}=z^{3}-\frac{3}{2}\left(x^{2}+y^{2}\right) z
$$

is solved. The mean value with the HFB intrinsic state $|\Phi\rangle$ of the two operators define the quadrupole and octupole deformation parameters $Q_{20}$ and $Q_{30}$. From them 

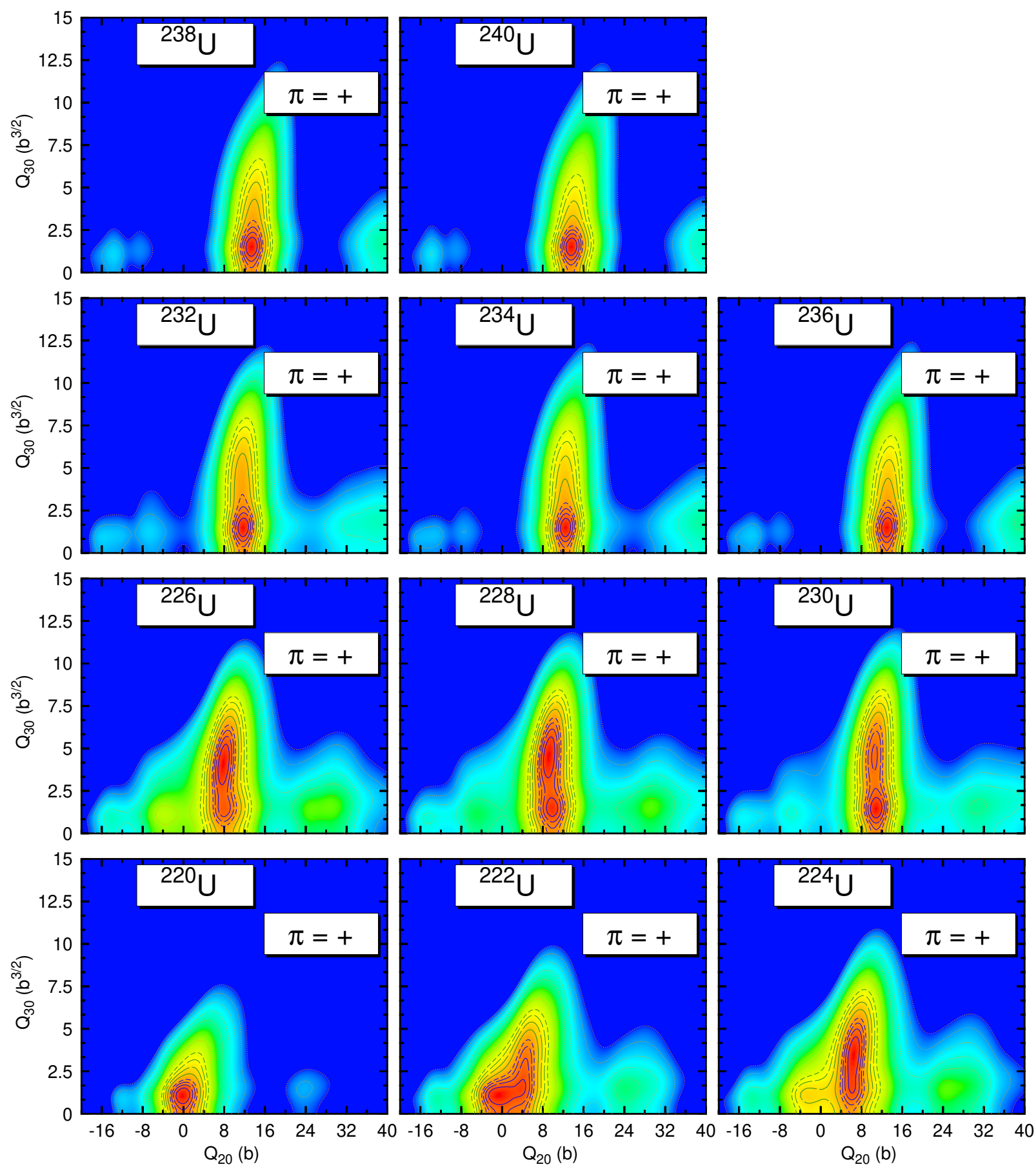

FIG. 3: (Color online) Positive $\pi=+1$ parity-projected potential energy surfaces (PPPESs) computed with the Gogny-D1M EDF for the isotopes ${ }^{220-240}$ U. See, caption of Fig. 1 for the contour-line patterns.

one can compute 35] the standard deformation parameters $\beta_{l}=\sqrt{4 \pi(2 l+1)} /\left(3 R_{0}^{l} A\right) Q_{l 0}$ with $R_{0}=1.2 A^{1 / 3}$ 1 In order to alleviate the already substantial com-

${ }^{1}$ For $A=230$ a value of $Q_{20}=1000 \mathrm{fm}^{2}$ is equivalent to $\beta_{2}=$ 0.212 and a value of $Q_{30}=1000 \mathrm{fm}^{3}$ is equivalent to $\beta_{3}=0.034$. putational effort, both axial and time-reversal symmetries have been kept as self-consistent symmetries. The HFB equation is solved using a performing, approximate second-order gradient method 64. The center of mass is fixed at the origin to avoid spurious effects associated with its motion [55, 57. The HFB quasiparticle operators [56] have been expanded in a deformed (axially symmetric) harmonic oscillator $(\mathrm{HO})$ basis containing 16 major 
shells to grant convergence for the studied physical quantities.

The $\left(Q_{20}, Q_{30}\right)$-constrained Gogny-HFB calculations provide a set of states $|\Phi(\mathbf{Q})\rangle$ labeled by their corresponding static deformations $\mathbf{Q}=\left(Q_{20}, Q_{30}\right)$. The HFB energies $E_{H F B}(\mathbf{Q})$ associated with those states define the contour plots referred to as MFPESs in this work. As the Gogny-EDF is invariant under parity transformation 65. 66 the associated HFB energies satisfy the property $E_{H F B}\left(Q_{20}, Q_{30}\right)=E_{H F B}\left(Q_{20},-Q_{30}\right)$. For this reason, only positive octupole moments are considered when plotting PESs.

The MFPESs obtained for the isotopes ${ }^{220-240} \mathrm{U}$ are shown in Fig. 1 as illustrative examples. In our calculations, the $Q_{20}$-grid $-20 \mathrm{~b} \leq Q_{20} \leq 40 \mathrm{~b}$ (with a step $\left.\delta Q_{20}=1 \mathrm{~b}\right)$ and the $Q_{30}$-grid $0 \mathrm{~b}^{3 / 2} \leq Q_{30} \leq 15 \mathrm{~b}^{3 / 2}$ (with a step $\delta Q_{30}=0.5 \mathrm{~b}^{3 / 2}$ ) have been employed. Along the $Q_{20}$-direction there is a shape/phase transition from a spherical ground state in ${ }^{220} \mathrm{U}$ to a well quadrupole deformed ground state in ${ }^{240} \mathrm{U}$. A similar structural evolution along the $Q_{20}$-direction have been obtained for the $\mathrm{Pu}, \mathrm{Cm}$, and $\mathrm{Cf}$ isotopic chains. Spherical or weakly deformed ground states are obtained for isotopes with $N \approx 126$ while a well quadrupole deformed ground state emerges with increasing neutron number. In fact, we have obtained (static) HFB quadrupole deformations within the range $0 \mathrm{~b} \leq Q_{20, G S} \leq 14 \mathrm{~b}$. The only exception is the nucleus ${ }^{222} \mathrm{Cf}$ for which $Q_{20, G S}=-2 \mathrm{~b}$. Many of the considered isotopes exhibit octupole deformation in their HFB ground state: ${ }^{222-230} \mathrm{U},{ }^{224-232} \mathrm{Pu},{ }^{226-234} \mathrm{Cm}$ and ${ }^{228-232} \mathrm{Cf}$ with values of the octupole moment in the range $2 \mathrm{~b}^{3 / 2} \leq Q_{30, G S} \leq 5 \mathrm{~b}^{3 / 2}$.

The MFPESs depicted in Fig. 1, as well as the ones obtained for the $\mathrm{Pu}, \mathrm{Cm}$ and $\mathrm{Cf}$ isotopic chains are rather soft along the $Q_{30}$-direction. This is further illustrated in Fig. 5 where the HFB energies obtained for ${ }^{220} \mathrm{U},{ }^{226} \mathrm{U}$ and ${ }^{234} \mathrm{U}$ have been plotted, as functions of $Q_{30}$, for fixed values of the quadrupole moment corresponding to the absolute minima of the PESs.

The mean-field octupole correlation energies defined as the energy gained by allowing octupolarity in the ground state

$$
\Delta E_{C O R R, H F B}=E_{H F B, Q_{30}=0}-E_{H F B, G S}
$$

are plotted in Fig. 2 The largest values $(1.25,1.04,0.81$ and $0.59 \mathrm{MeV}$ ) correspond to $N=134$ isotones. Note, that the relatively small energies $E_{C O R R, H F B}$ result from the softness observed in the MFPESs of nuclei with octupole deformed ground states [see, for example, panel (b) of Fig. 5 .

The softness of the MFPESs discussed in this section already point towards the key role of dynamical beyond-mean-field correlations, i.e., symmetry restoration and/or quadrupole-octupole configuration mixing in the studied nuclei. Two spatial symmetries are broken in this study. One is the rotational symmetry with the quadrupole moment as the relevant parameter and the other is the reflection symmetry with the octupole moment as the relevant parameter. From the previous discussion of mean-field results it is clear that the octupole is the softest mode. Therefore, parity is the most important symmetry to be restored. It would be desirable to restore also both the rotational and particle number symmetries. This kind of simultaneous symmetry restoration is feasible in lighter nuclear systems. However, when combined with the quadrupole-octupole configuration mixing of Sec. IIC, it becomes a highly demanding computational task [43] out of the scope of an exhaustive survey like the one discussed in this paper.

\section{B. Parity symmetry restoration}

Parity symmetry is broken by intrinsic HFB states with a non-zero value of the octupole moment. To restore the symmetry [23, 32, 55] we build parity-projected states $\left|\Phi^{\pi}(\mathbf{Q})\right\rangle$ from the intrinsic HFB states $|\Phi(\mathbf{Q})\rangle$ by acting on them with the parity projector

$$
\hat{\mathcal{P}}^{\pi}=\frac{1}{2}(1+\pi \hat{\Pi}),
$$

where $\pi= \pm 1$ is the desired parity quantum number. For each of the projected states with parity $\pi$ one can compute the projected energy

$$
\begin{aligned}
E_{\pi}(\mathbf{Q}) & =\frac{\langle\Phi(\mathbf{Q})|\hat{H}[\rho(\vec{r})]| \Phi(\mathbf{Q})\rangle}{\langle\Phi(\mathbf{Q}) \mid \Phi(\mathbf{Q})\rangle+\pi\langle\Phi(\mathbf{Q})|\hat{\Pi}| \Phi(\mathbf{Q})\rangle} \\
& +\pi \frac{\langle\Phi(\mathbf{Q})|\hat{H}[\theta(\vec{r})] \hat{\Pi}| \Phi(\mathbf{Q})\rangle}{\langle\Phi(\mathbf{Q}) \mid \Phi(\mathbf{Q})\rangle+\pi\langle\Phi(\mathbf{Q})|\hat{\Pi}| \Phi(\mathbf{Q})\rangle}
\end{aligned}
$$

The evaluation of the Hamiltonian overlaps $\langle\Phi(\mathbf{Q})|\hat{H}[\rho(\vec{r})]| \Phi(\mathbf{Q})\rangle \quad$ and $\quad\langle\Phi(\mathbf{Q})|\hat{H}[\theta(\vec{r})] \hat{\Pi}| \Phi(\mathbf{Q})\rangle \quad$ in Eq. (5) requires a prescription for the density-dependent part of the Gogny-EDF. As in previous studies [55, 57, we use the mixed density prescription that amounts to consider the densities

$$
\rho(\vec{r})=\frac{\langle\Phi(\mathbf{Q})|\hat{\rho}(\vec{r})| \Phi(\mathbf{Q})\rangle}{\langle\Phi(\mathbf{Q}) \mid \Phi(\mathbf{Q})\rangle}
$$

and

$$
\theta(\vec{r})=\frac{\langle\Phi(\mathbf{Q})|\hat{\rho}(\vec{r}) \hat{\Pi}| \Phi(\mathbf{Q})\rangle}{\langle\Phi(\mathbf{Q})|\hat{\Pi}| \Phi(\mathbf{Q})\rangle}
$$

Such a prescription guarantees various consistency requirements within the EDF framework and avoids pathologies associated with the restoration of spatial symmetries 65 68. The parity-projected proton and neutron numbers, usually differ from the nucleus' proton $Z_{0}$ and neutron $N_{0}$ numbers. To correct the energy for this deviation we have replaced $\hat{H}$ by $\hat{H}-\lambda_{Z}\left(\hat{Z}-Z_{0}\right)-$ $\lambda_{N}\left(\hat{N}-N_{0}\right)$, where $\lambda_{Z}$ and $\lambda_{N}$ are chemical potentials for protons and neutrons, respectively [55, 57, 69, 70. 

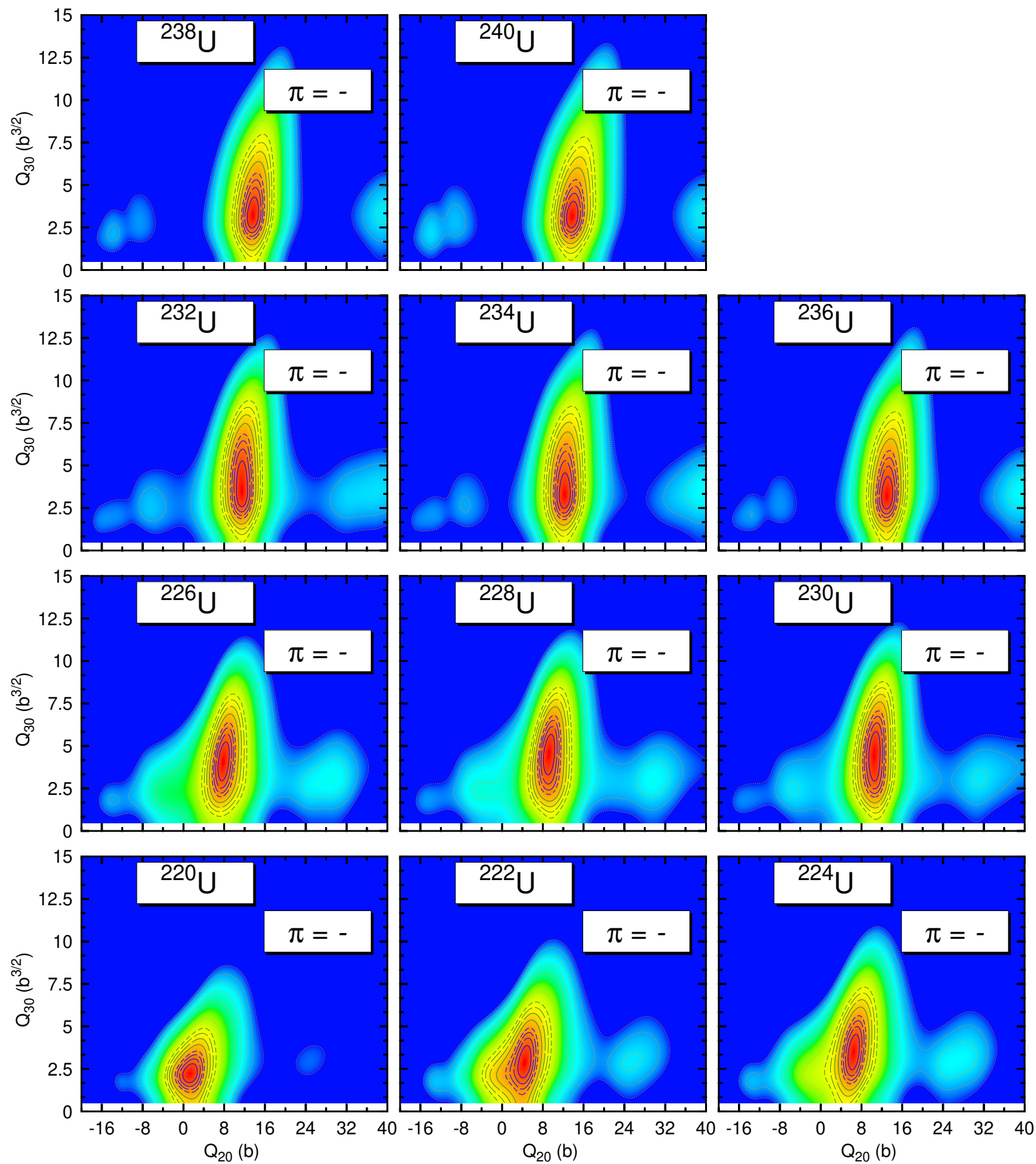

FIG. 4: (Color online) Negative $\pi=-1$ parity-projected potential energy surfaces (PPPESs) computed with the Gogny-D1M EDF for the isotopes ${ }^{220-240}$ U. See, caption of Fig. 1 for the contour-line patterns.

The $\pi=+1$ and $\pi=-1$ PPPESs obtained for the isotopes ${ }^{220-240} \mathrm{U}$ are depicted in Figs. 3 and 4 as illustrative examples. Along the $Q_{30}=0$ axis, the projection onto positive parity is unnecessary as the corresponding quadrupole deformed even-even intrinsic states are already pure $\pi=+1$ states. On the other hand, in the case of negative parity, the evaluation of the projected energy along the $Q_{30}=0$ axis requires to resolve a "zero-over-zero" indeterminacy [32, 55. However, the $\pi=-1$ projected energy increases rapidly when approaching $Q_{30}=0$ (see, Fig. 5) and its limiting value does not play a significant role in the discussion of the PPPESs. We have then omitted this quantity along the $Q_{30}=0$ axis in Fig. 4.

The absolute minima of the $\pi=+1$ and $\pi=-1$ PPPESs are located at quadrupole deformations close 

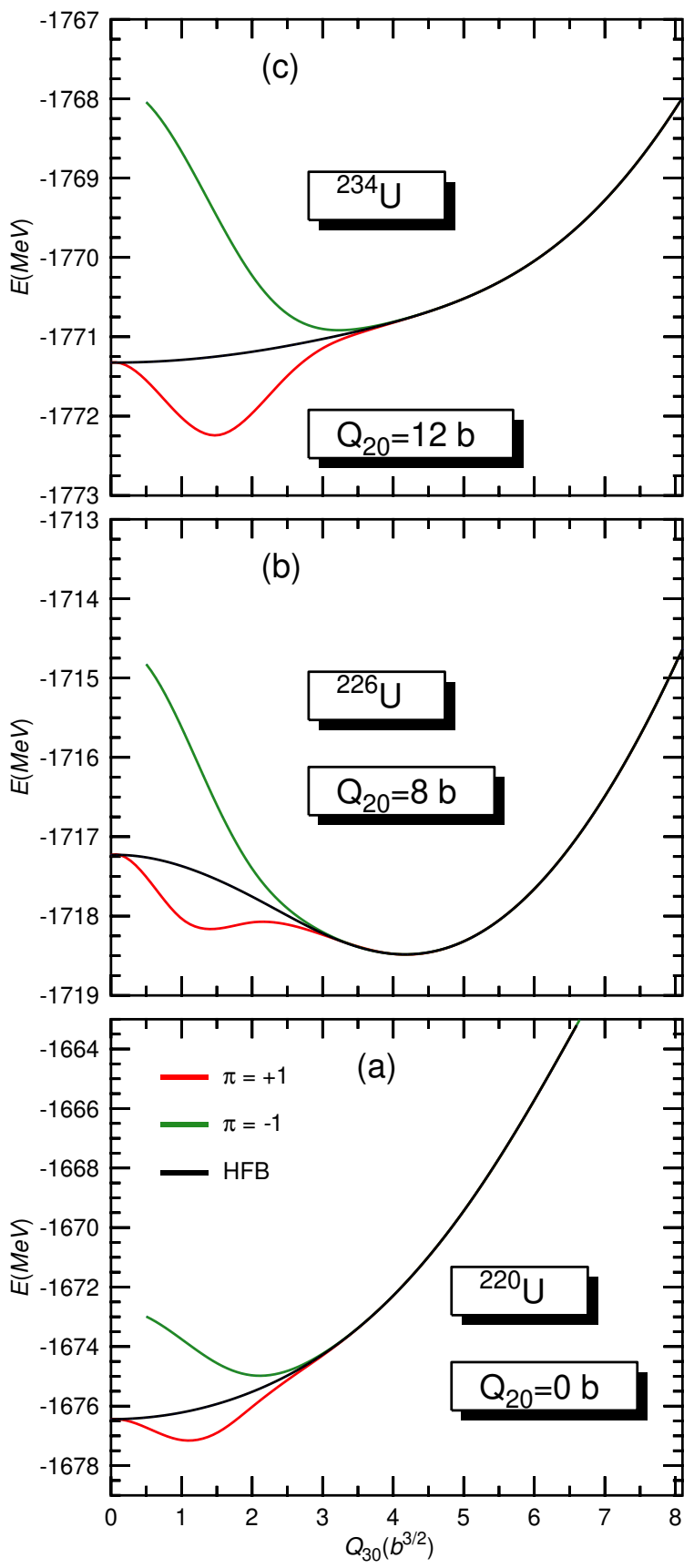

FIG. 5: (Color online) The $\pi=+1$ (red) and $\pi=-1$ (green) parity-projected energies are depicted as functions of the octupole moment $Q_{30}$ for fixed values of the quadrupole moment $Q_{20}$ in the nuclei ${ }^{220} \mathrm{U},{ }^{226} \mathrm{U}$ and ${ }^{234} \mathrm{U}$. The corresponding HFB energies are also included in the plots. Results have been obtained with the Gogny-D1M EDF.

to the HFB values discussed in Sec. IIA. In the case of the $\pi=+1$ PPPESs, depicted in Fig. 3, a characteristic pocket develops with a minimum at $Q_{30}=1.0-1.5 b^{3 / 2}$. In the case of nuclei with a reflection-symmetric HFB ground state, such a minimum is the global one. This is illustrated in panels (a) and (c) of Fig. 5 where the $\pi=+1$ parity-projected energies obtained for ${ }^{220} \mathrm{U}$ and

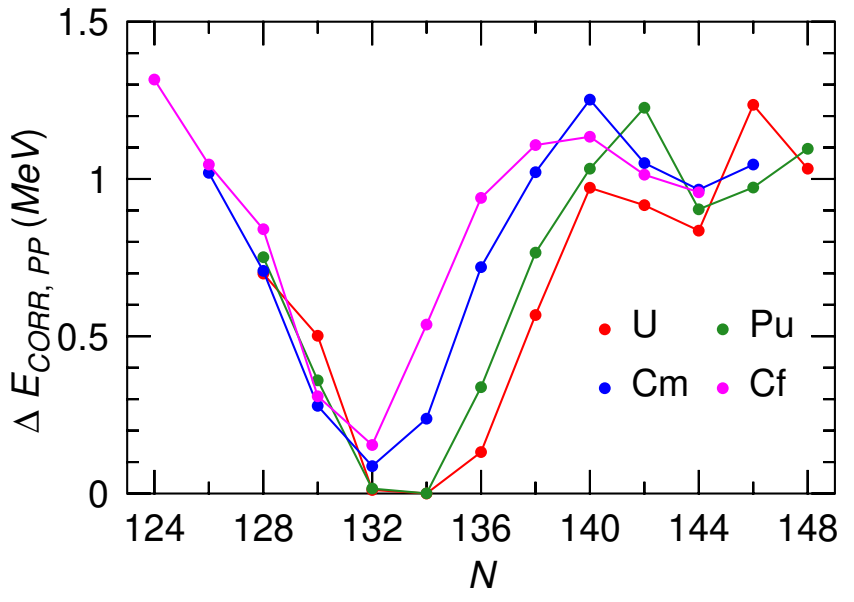

FIG. 6: (Color online) The correlation energies stemming from parity restoration Eq.8 are plotted as functions of the neutron number. Results have been obtained with the GognyD1M EDF. For more details, see the main text.

${ }^{234} \mathrm{U}$ are plotted, as functions of $Q_{30}$, for fixed values of the quadrupole moment corresponding to the absolute minima of the PESs. On the other hand, for nuclei with a reflection-asymmetric mean-field ground state, there is a pronounced competition with a second minimum at $Q_{30}=3.0-4.5 b^{3 / 2}$ as illustrated in panel (b) of Fig. 5 for ${ }^{226} \mathrm{U}$. In the case of ${ }^{226} \mathrm{U}$, the global $\pi=+1$ minimum at $Q_{30}=4.0 b^{3 / 2}$ is only $500 \mathrm{KeV}$ deeper than the one at $Q_{30}=1.0 b^{3 / 2}$. Similar results have been obtained for $\mathrm{Pu}, \mathrm{Cm}$ and $\mathrm{Cf}$ isotopes. For example, the global $\pi=+1$ minima correspond to $Q_{30}=3.0 b^{3 / 2}$ and $4.0 b^{3 / 2}$ in ${ }^{226} \mathrm{Pu}$ and ${ }^{228} \mathrm{Pu}$, respectively, while for other $\mathrm{Pu}$ isotopes as well as for $\mathrm{Cm}$ and $\mathrm{Cf}$ nuclei they are located at $Q_{30}=1.0-1.5 b^{3 / 2}$. As can be seen from Figs. 1, 3 and 5 not only the MFPESs but also the $\pi=+1$ PPPESs are rather soft along the $Q_{30}$-direction.

The $\pi=-1$ PPPESs, depicted in Fig. 4, display well developed absolute minima at $Q_{30}=2.0-4.5 b^{3 / 2}$. In the case of nuclei with a reflection-symmetric HFB ground state, such as ${ }^{220} \mathrm{U}$ and ${ }^{234} \mathrm{U}$, the absolute $\pi=-1$ minima have larger octupole deformations than the $\pi=+1$ ones [see, panels (a) and (c) of Fig. 5. On the other hand, for some nuclei with a reflection-asymmetric HFB ground state, such as ${ }^{226} \mathrm{U}$, the (almost degenerate) $\pi=-1$ and $\pi=+1$ absolute minima have similar octupole deformations [see, panel (b) of Fig. 5. Similar features have been found for the other isotopic chains. Let us mention, that the complex topography along the $Q_{30}$-direction as well as the transition to an octupole-deformed regime found in our Gogny-D1M calculations has also been studied, as a function of the strength of the two-body interaction, in Ref. [71 using the parity-projected Lipkin-MeshkovGlick (LMG) model.

As a measure of the correlations induced by parity symmetry restoration one can use the correlation energy, defined in terms of the difference between the HFB $E_{H F B, G S}$ and parity projected $E_{\pi=+1, G S}$ ground state 

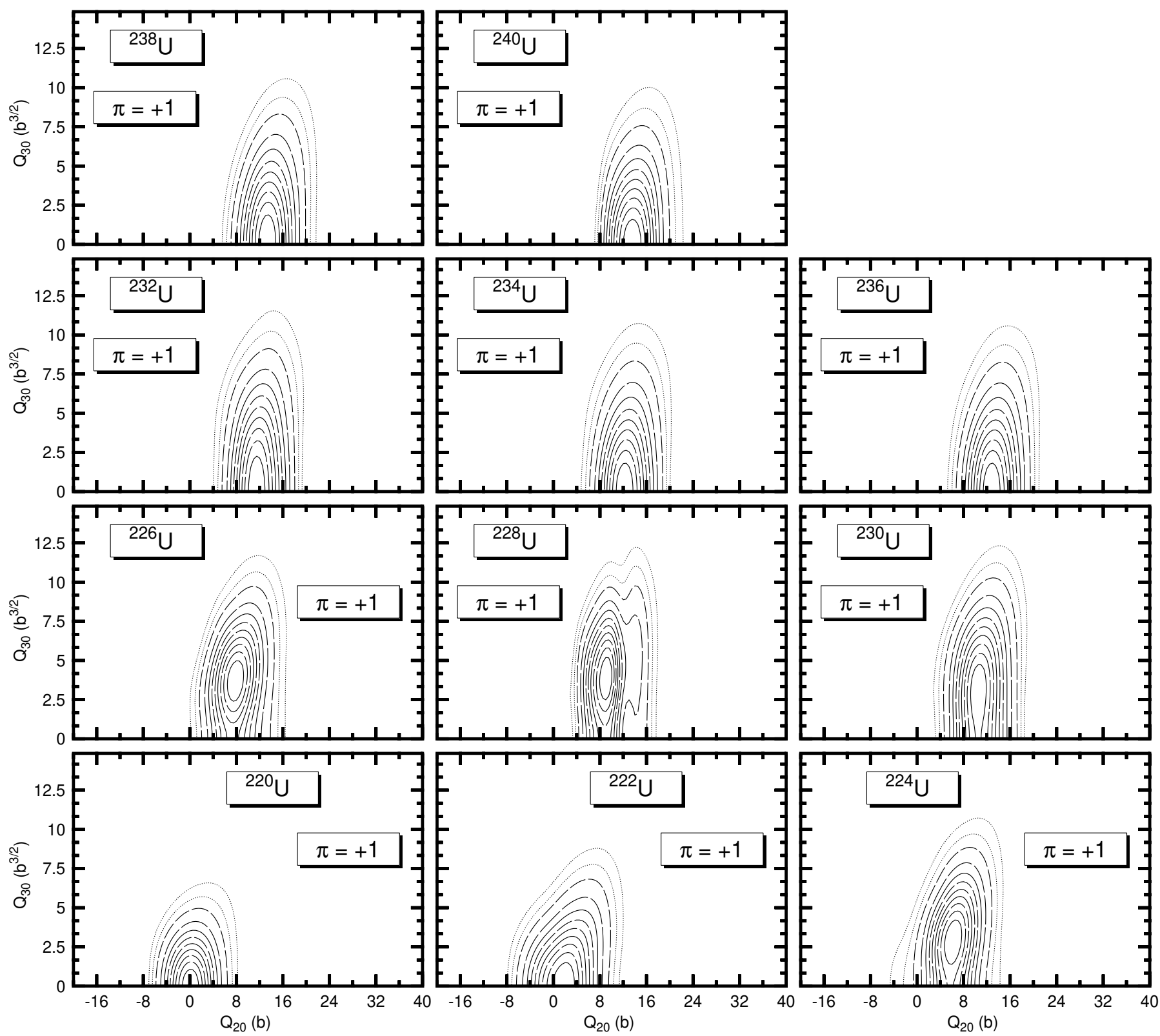

FIG. 7: Collective wave functions Eq. 13. squared for the ground states of the nuclei ${ }^{220-240} \mathrm{U}$. The contour lines (a succession of solid, long dashed and short dashed lines) start at $90 \%$ of the maximum value up $10 \%$ of it. The two dotted-line contours correspond to the tail of the amplitude ( $15 \%$ and $1 \%$ of the maximum value). Results have been obtained with the Gogny-D1M EDF. For more details, see the main text.

energies

$$
\Delta E_{C O R R, P P}=E_{H F B, G S}-E_{\pi=+1, G S} .
$$

In Fig. 6, we show this quantity for the different isotopes considered. The correlation energy shows a minimum around $N=132-134$ corresponding to strongly octupole-deformed intrinsic states. As shown later on in Sec. IIC the comparison between the correlation energies $E_{C O R R, P P}$ and the ones obtained within the symmetryconserving 2D-GCM framework (see, Fig. 9 reveals the key role played by quantum fluctuations around those neutron numbers.

\section{Generator Coordinate Method}

We include quantum fluctuations in the quadrupole and octupole degrees of freedom by considering a linear superposition of the HFB states $|\Phi(\mathbf{Q})\rangle$

$$
\left|\Psi_{\sigma}^{\pi}\right\rangle=\int d \mathbf{Q} f_{\sigma}^{\pi}(\mathbf{Q})|\Phi(\mathbf{Q})\rangle
$$

where, both positive and negative octupole moments $Q_{30}$ are included in the integration domain. In this way the parity of the collective amplitude under the change of sign 

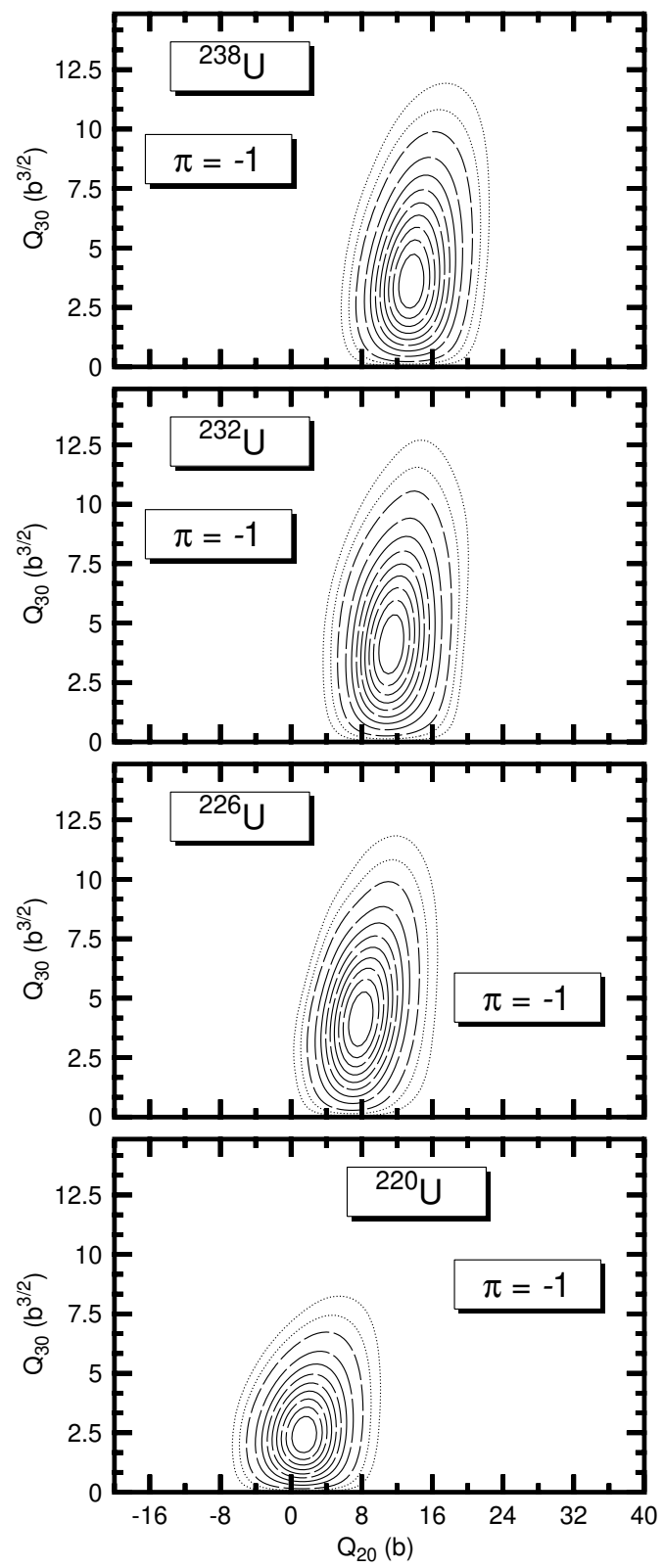
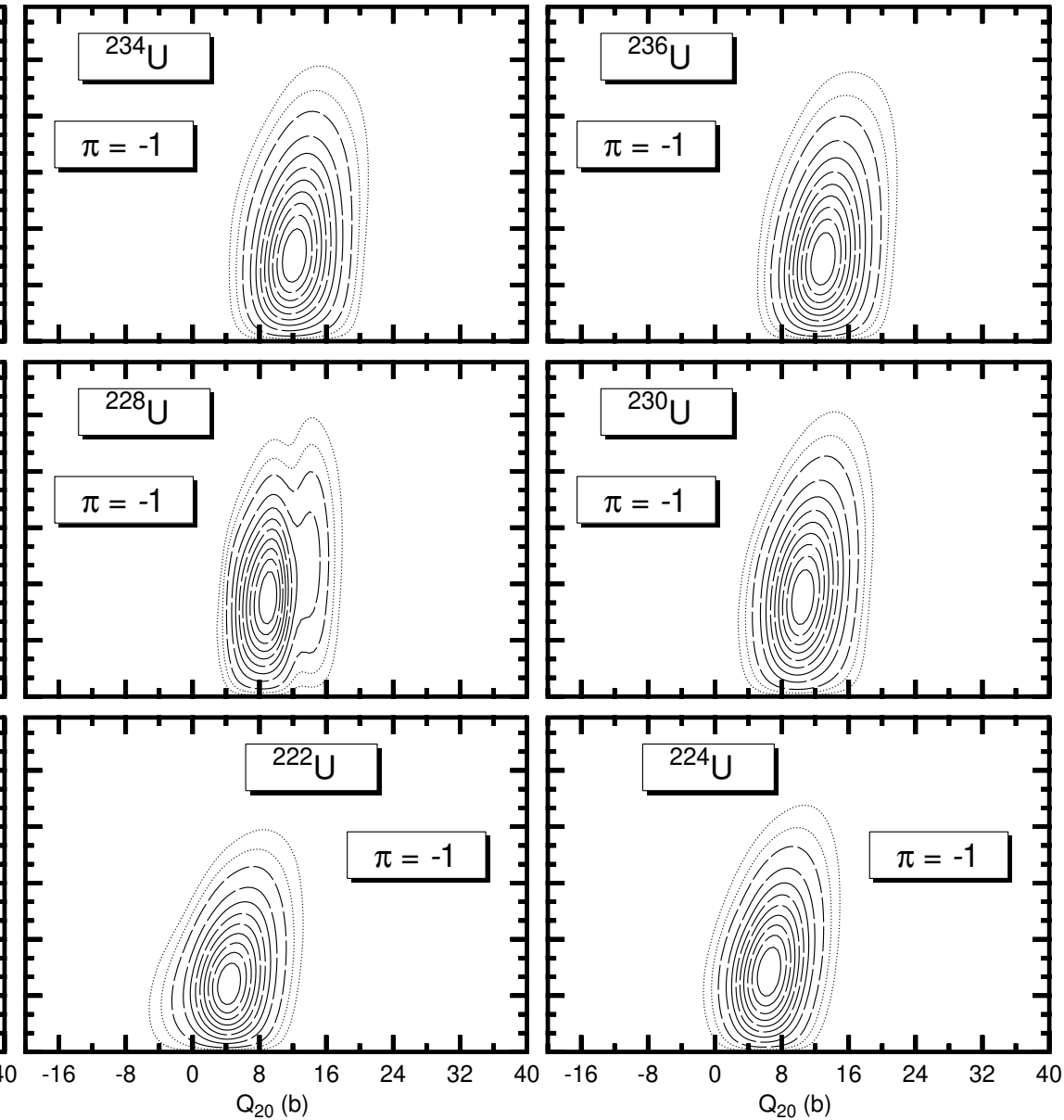

FIG. 8: Collective wave functions Eq. 13 squared for the lowest negative-parity states of the nuclei ${ }^{220-240} \mathrm{U}$. See, caption of Fig. 7 for contour-line patterns. Results have been obtained with the Gogny-D1M EDF. For more details, see the main text.

of $Q_{3}$, namely $f_{\sigma}^{\pi}\left(Q_{20},-Q_{30}\right)=\pi f_{\sigma}^{\pi}\left(Q_{20}, Q_{30}\right)$, determines the parity of $\left|\Psi_{\sigma}^{\pi}\right\rangle$. The property $f_{\sigma}^{\pi}\left(Q_{20},-Q_{30}\right)=$ $\pi f_{\sigma}^{\pi}\left(Q_{20}, Q_{30}\right)$ is a direct consequence of the invariance of the interaction under the parity symmetry operation. The index $\sigma$ in Eq. (9) labels the different GCM solutions.

The amplitudes $f_{\sigma}^{\pi}(\mathbf{Q})$ are solutions of the Griffin-HillWheeler (GHW) equation 56.

$$
\int d \mathbf{Q}^{\prime}\left(\mathcal{H}\left(\mathbf{Q}, \mathbf{Q}^{\prime}\right)-E_{\sigma}^{\pi} \mathcal{N}\left(\mathbf{Q}, \mathbf{Q}^{\prime}\right)\right) f_{\sigma}^{\pi}\left(\mathbf{Q}^{\prime}\right)=0 .
$$

with the Hamiltonian and norm kernels defined in the standard way

$$
\mathcal{H}\left(\mathbf{Q}, \mathbf{Q}^{\prime}\right)=\left\langle\Phi(\mathbf{Q})\left|\hat{H}\left[\rho^{G C M}(\vec{r})\right]\right| \Phi\left(\mathbf{Q}^{\prime}\right)\right\rangle
$$

$$
\mathcal{N}\left(\mathbf{Q}, \mathbf{Q}^{\prime}\right)=\left\langle\Phi(\mathbf{Q}) \mid \Phi\left(\mathbf{Q}^{\prime}\right)\right\rangle
$$

In the evaluation of the Hamiltonian kernel $\mathcal{H}\left(\mathbf{Q}, \mathbf{Q}^{\prime}\right)$ for the Gogny-EDF, we have employed the mixed density prescription

$$
\rho^{G C M}(\vec{r})=\frac{\left\langle\Phi(\mathbf{Q})|\hat{\rho}(\vec{r})| \Phi\left(\mathbf{Q}^{\prime}\right)\right\rangle}{\left\langle\Phi(\mathbf{Q}) \mid \Phi\left(\mathbf{Q}^{\prime}\right)\right\rangle} .
$$

As in the parity projection case, first-order corrections to take into account deviations in both the proton and neutron numbers [55, 57, 69, 70 are included.

The HFB basis states $|\Phi(\mathbf{Q})\rangle$ are not orthonormal. Therefore, the amplitudes $f_{\sigma}^{\pi}(\mathbf{Q})$ cannot be interpreted 


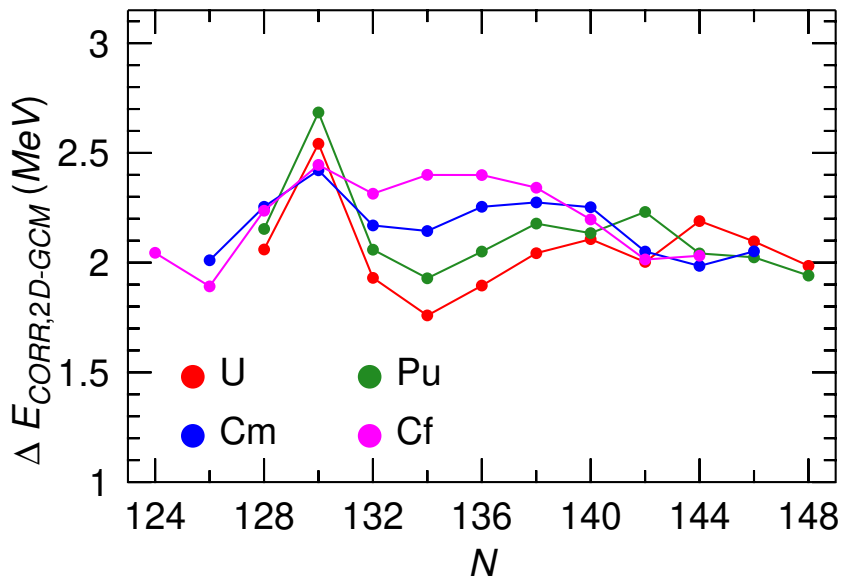

FIG. 9: (Color online) The correlation energies obtained within the 2D-GCM framework Eq. 20 are plotted as functions of the neutron number. Results have been obtained with the Gogny-D1M EDF. For more details, see the main text.

as probability amplitudes. Instead, one considers the socalled collective wave functions

$$
G_{\sigma}^{\pi}(\mathbf{Q})=\int d \mathbf{Q}^{\prime} \mathcal{N}^{\frac{1}{2}}\left(\mathbf{Q}, \mathbf{Q}^{\prime}\right) f_{\sigma}^{\pi}\left(\mathbf{Q}^{\prime}\right)
$$

written in terms of the square root operator $\mathcal{N}^{\frac{1}{2}}\left(\mathbf{Q}, \mathbf{Q}^{\prime}\right)$ of the norm kernel [55, 56, 65] defined by the property

$$
\mathcal{N}\left(\mathbf{Q} ; \mathbf{Q}^{\prime}\right)=\int d \mathbf{Q}^{\prime \prime} \mathcal{N}^{\frac{1}{2}}\left(\mathbf{Q} ; \mathbf{Q}^{\prime \prime}\right) \mathcal{N}^{\frac{1}{2}}\left(\mathbf{Q}^{\prime \prime} ; \mathbf{Q}^{\prime}\right)
$$

The overlap $\left\langle\Psi_{\sigma}^{\pi}|\hat{O}| \Psi_{\sigma^{\prime}}^{\pi^{\prime}}\right\rangle$ of an operator $\hat{O}$ between two different GCM states Eq. (9) is required in the computation of physical quantities such as, for example, the electromagnetic transition probabilities. It reads

$$
\left\langle\Psi_{\sigma}^{\pi}|\hat{O}| \Psi_{\sigma^{\prime}}^{\pi^{\prime}}\right\rangle=\int d \mathbf{Q} d \mathbf{Q}^{\prime} G_{\sigma}^{\pi *}(\mathbf{Q}) \mathcal{O}\left(\mathbf{Q}, \mathbf{Q}^{\prime}\right) G_{\sigma^{\prime}}^{\pi^{\prime}}\left(\mathbf{Q}^{\prime}\right)
$$

where

$$
\begin{aligned}
\mathcal{O}\left(\mathbf{Q}, \mathbf{Q}^{\prime}\right) & =\int d \mathbf{Q}^{\prime \prime} d \mathbf{Q}^{\prime \prime \prime} \mathcal{N}^{-\frac{1}{2}}\left(\mathbf{Q} ; \mathbf{Q}^{\prime \prime}\right)\left\langle\mathbf{Q}^{\prime \prime}|\hat{O}| \mathbf{Q}^{\prime \prime \prime}\right\rangle \times \\
& \times \mathcal{N}^{-\frac{1}{2}}\left(\mathbf{Q}^{\prime \prime \prime} ; \mathbf{Q}^{\prime}\right)
\end{aligned}
$$

For the reduced transition probabilities $B\left(E 1,1^{-} \rightarrow\right.$ $\left.0^{+}\right)$and $B\left(E 3,3^{-} \rightarrow 0^{+}\right)$the rotational formula for $\mathrm{K}=0$ bands have been used

$$
B\left(E \lambda, \lambda^{-} \rightarrow 0^{+}\right)=\frac{e^{2}}{4 \pi}\left|\left\langle\Psi_{\sigma}^{\pi=-1}\left|\hat{\mathcal{O}}_{\lambda}\right| \Psi_{\sigma^{\prime}=1}^{\pi^{\prime}=+1}\right\rangle\right|^{2} .
$$

For $B(E 1)$ and $B(E 3)$ transitions $\sigma$ corresponds to the first excited GCM state with negative parity. The electromagnetic transition operators $\hat{\mathcal{O}}_{1}$ and $\hat{\mathcal{O}}_{3}$ are the dipole moment operator and the proton component of the octupole operator, respectively [55].

Some comments are in order here regarding the use of Eq.[17]. Previous studies [72, 73] have revealed that the use of proper angular momentum projected (AMP) wave functions concurs in an enhancement of the $E 3$ strengths in spherical and/or weakly quadrupole deformed nuclei as compared to the strength obtained with the rotational formula implicit in Eq.(17). On the other hand, the E1 transitions do not show a clear pattern due to their less collective nature. With this in mind, the $E 3$ strengths obtained in our calculations for spherical and/or weakly deformed $N \approx 126$ nuclei via Eq.(17), should be viewed as lower bounds.

The collective wave functions Eq.13 squared corresponding to the ground and lowest negative parity 2DGCM states in ${ }^{220-240} \mathrm{U}$ are plotted in Figs. 7 and 8 , respectively. As can be seen from Fig. 7, the ground state collective amplitudes $\left|G_{\sigma=1}^{\pi=+1}\left(Q_{20}, Q_{30}\right)\right|^{2}$ reach global maxima for octupole moments different from zero only in ${ }^{224-230} \mathrm{U}$. The same holds for ${ }^{226-232} \mathrm{Pu}$ and ${ }^{228,230} \mathrm{Cm}$ while for other $\mathrm{U}, \mathrm{Pu}$ and $\mathrm{Cf}$ nuclei, the peaks are located around $Q_{30}=0$. As illustrated in Fig. 7. the spreading of the amplitudes $\left|G_{\sigma=1}^{\pi=+1}\left(Q_{20}, Q_{30}\right)\right|^{2}$ along the $Q_{30^{-}}$ direction is large, indicating the octupole-soft character of the $\pi=+12 \mathrm{D}-\mathrm{GCM}$ ground states. In the case of the $\pi=-1$ amplitudes, depicted in Fig. 8, the maxima are always located at a nonzero octupole moment as could be anticipated from the behavior of the $\pi=-1$ PPPESs (see, Fig. 4).

Using Eq.(15), we have computed the 2D-GCM average quadrupole moments

$$
\left(\bar{Q}_{20}\right)_{\sigma}^{\pi}=\left\langle\Psi_{\sigma}^{\pi}\left|\hat{Q}_{20}\right| \Psi_{\sigma}^{\pi}\right\rangle
$$

In the case of a negative-parity operator like $\hat{Q}_{30}$ the quantity $\left\langle\Psi_{\sigma}^{\pi}\left|\hat{Q}_{30}\right| \Psi_{\sigma}^{\pi}\right\rangle$ is zero by construction. Therefore, a meaningful averaged quantity has to be defined [55] by restricting the integration domain $\mathcal{D}$ to positive values of $Q_{30}$ and $Q_{30}^{\prime}$

$$
\left(\bar{Q}_{30}\right)_{\sigma}^{\pi}=4 \int_{\mathcal{D}} d \mathbf{Q} d \mathbf{Q}^{\prime} G_{\sigma}^{\pi *}(\mathbf{Q}) \mathcal{Q}_{30}\left(\mathbf{Q}, \mathbf{Q}^{\prime}\right) G_{\sigma}^{\pi}\left(\mathbf{Q}^{\prime}\right)
$$

In the case of a strongly peaked collective inertia, the average octupole moment $\bar{Q}_{30}$ is a good estimator of the location of the peak.

The ground-state dynamical quadrupole moments $\left(\bar{Q}_{20}\right)_{\sigma=1}^{\pi=+1}$ increase as more neutrons are added along a given isotopic chain and their values remain close to the ones predicted at the HFB level. On the other hand, at variance with the HFB results, once both $\pi=+1$ symmetry restoration and $\left(Q_{20}, Q_{30}\right)$-fluctuations are considered at the 2D-GCM level, dynamical octupole deformations $0.53 b^{3 / 2} \leq\left(\bar{Q}_{30}\right)_{\sigma=1}^{\pi=+1} \leq 2.15 b^{3 / 2}$ are found in the ground states of all the studied nuclei with the largest values corresponding to isotopes with neutron numbers $N=132-138$. The quadrupole moments $\left(\bar{Q}_{20}\right)_{\sigma}^{\pi=-1}$ corresponding to the lowest negative-parity states also increase their values with increasing $N$. Moreover, the corresponding average octupole moments lie within the range $1.87 b^{3 / 2} \leq\left(\bar{Q}_{30}\right)_{\sigma}^{\pi=-1} \leq 3.75 b^{3 / 2}$ with their largest values being reached once more for $N=132-138$ isotopes. 

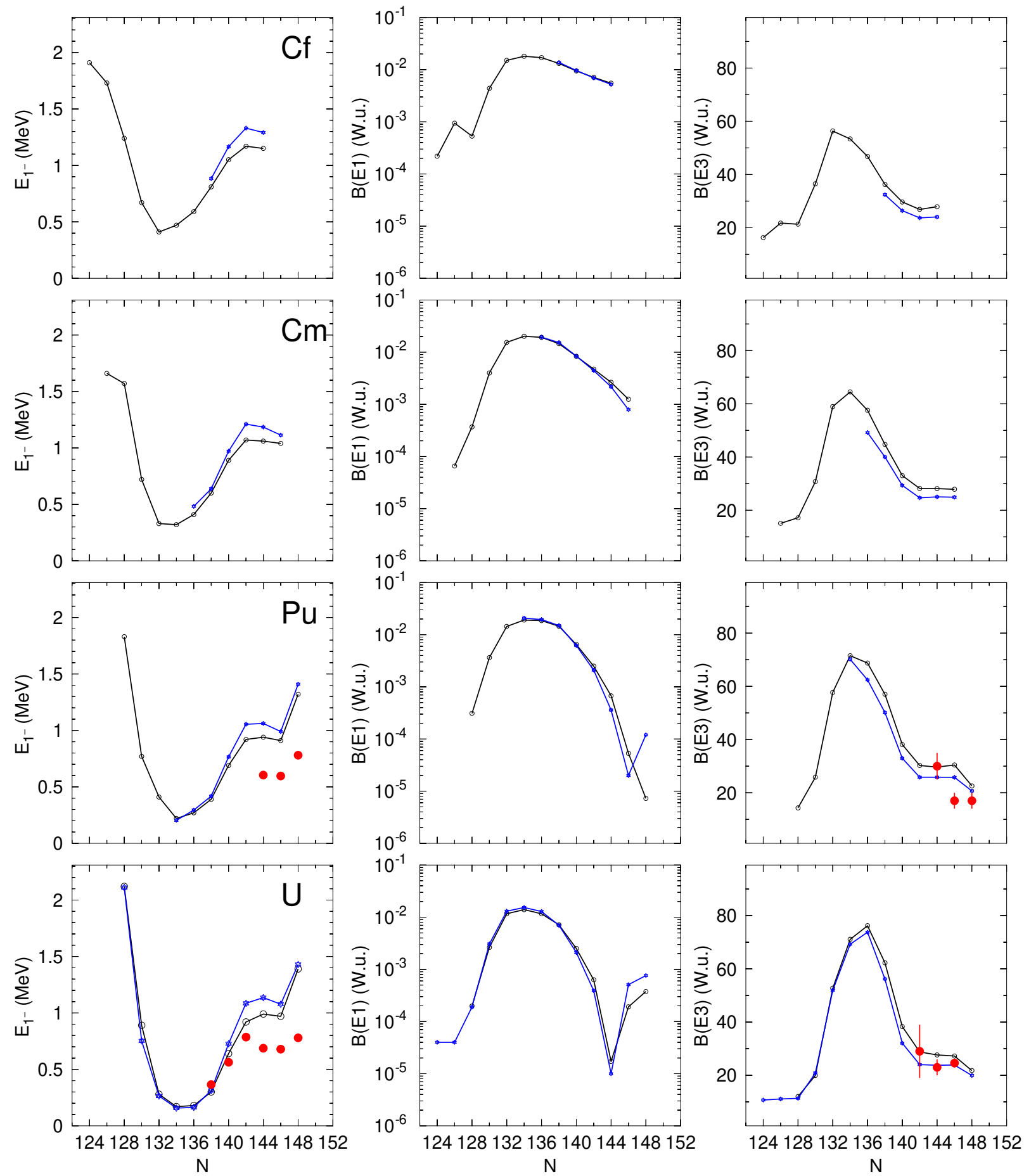

FIG. 10: (Color online) The 2D-GCM $E_{1}$ - energy splittings (left panels) and the reduced transition probabilities $B(E 1)$ (middle panels) and $B(E 3)$ (right panels) are plotted (in black) as functions of the neutron number for the studied $\mathrm{U}, \mathrm{Pu}, \mathrm{Cm}$ and $\mathrm{Cf}$ isotopic chains. The available experimental data (in red) have been taken from Ref. 63. The $E_{1^{-}}, B(E 1)$ and $B(E 3)$ values obtained in the framework of the 1D-GCM [53], with the octupole moment as single generating coordinate, have also been included (in blue) in each of the plots. Results have been obtained with the Gogny-D1M EDF. For more details, see the main text. 
The correlation energies, defined as the difference between the HFB and 2D-GCM ground-state energies

$$
\Delta E_{C O R R, 2 D-G C M}=E_{H F B, G S}-E_{\pi=+1,2 D-G C M}
$$

are depicted in Fig. 9. They exhibit a weaker dependence with neutron number than the $\Delta E_{C O R R, P P}$ values stemming from parity restoration (see, Fig. 6. The inclusion of beyond-mean-field correlations, via the 2D-GCM ansatz Eq. (9), substantially modifies the behavior observed in Fig. 6 around the neutron numbers $N=132-134$ providing a smoother trend. Furthermore, the variation of the correlation energies (within the range $1.76 \mathrm{MeV} \leq \Delta E_{C O R R, 2 D-G C M} \leq 2.46 \mathrm{MeV}$ ) is of the same order of magnitude as the rms for the binding energy in Gogny-like nuclear mass tables [4] and, therefore, those correlation energies should be considered in improved versions of the Gogny-EDF.

The energy difference $E_{1}$ - between the positive parity ground state and the lowest $1^{-}$excited state, obtained in the 2D-GCM calculations, is shown in the left panels of Fig. 10 as a function of the neutron number. The energies are very small for ${ }^{224-230} \mathrm{U}$ in agreement with their large (dynamical) octupole deformation. Other U isotopes, with less pronounced dynamical octupole deformation effects, display larger $E_{1^{-}}$values and the first negative parity excited state can be interpreted as an octupole vibrational state. In the same panels, we have also included the energy differences $E_{1-}$ obtained within the framework of the 1D-GCM with the octupole moment as single generating coordinate [53. As can be seen, the trend with neutron number is similar in both calculations. However, for heavier isotopes the 2D-GCM $E_{1}$ - energies tend to be smaller than the 1D-GCM ones. Regarding the comparison with the the available experimental data, we are able to reproduce the increase of the excitation energies with increasing neutron number. However, exception made of the $N=138-140$ isotopes, the predicted $E_{1-}$ energies are larger than the experimental ones, a feature found in many GCM calculations (see, for example, 53, 55]). Similar results are found for the other isotopic chains.

In the case of the $B(E 1)$ reduced transition probabilities, depicted in the middle panels of Fig. 10, no experimental data are available. Exception made of the nucleus ${ }^{242} \mathrm{Pu}$, the $1 \mathrm{D}-\mathrm{GCM}$ and 2D-GCM calculations display a similar pattern with the largest $B(E 1)$ values corresponding to the neutron numbers $N=132-136$. As discussed in 31, the $B(E 1)$ strength strongly depend on how the dipole moment evolves with octupole deformation in the region where the positive and negative parity wave functions overlap. In the ${ }^{242} \mathrm{Pu}$ case the dipole moment changes sign in the region of interest and there is a strong cancellation depending upon subtle details of the collective wave functions. For other nuclei, however, the sign of the dipole moment does not change with octupole deformation and the dependency with the details of the collective wave functions is much weaker. Although the 1D and 2D GCM collective wave functions look very similar, the tiny differences can easily explain the differences in the results of the two calculations. Note, that both approaches predict a pronounced minimum for ${ }^{236} \mathrm{U}$ also consequence of a dipole moment changing its sign as the octupole moment increases. The $B(E 3)$ reduced transition probabilities are plotted in the right panels of Fig. 10. They show marked maxima for $N=132-136$ that correlate well with the features observed for the $E_{1-}$ energies and the $B(E 1)$ strengths. Though essentially the same trend is obtained, for heavier nuclei the 2DGCM $B(E 3)$ values are larger than the 1D-GCM ones. As can be seen from the panels, the predicted $B(E 3)$ strengths for ${ }^{234-238} \mathrm{U}$ and ${ }^{238-242} \mathrm{Pu}$ compare reasonably well with the available experimental data.

Finally, let us mention that the comparison between the 2D-GCM and 1D-GCM results in Fig. 10 reveals that, to a large extent, there is a decoupling between the quadrupole and octupole degrees of freedom in the studied nuclei and confirms that the 1D-GCM approach 53. represents a valuable computational tool to account for the systematic of the $1^{-}$energy splittings and reduced transition probabilities in this region of the nuclear chart.

In order to explore the robustness of the results with a change of the parametrization of the interaction, we have carried out in the uranium chain the same kind of 2D GCM calculations but with the D1S and D1M* parametrizations of the Gogny force. The later is a newly proposed re-parametrization of D1M with the goal of improving the slope of the symmetry energy [74] while preserving as much as possible other properties of D1M. The results are shown in Fig 11 along with the experimental data.

The trend with neutron number is similar in the three calculations confirming the consistency of the results. There are some quantitative differences at $N=130$ where a transition from octupole soft to octupole deformed ground state takes place. Those differences are larger for D1S as expected, because D1M* was fitted to be as close as possible to D1M. From the comparison we conclude that the trend of the results with neutron number is rather insensitive to the interaction used.

\section{CONCLUSIONS}

In this paper we have studied the interplay between the quadrupole and octupole degrees of freedom in a selected set of even-even actinides both at the mean-field level and beyond. To this end, we have resorted to the static Gogny-HFB approach, parity projection as well as 2DGCM calculations with the multipole moments $Q_{20}$ and $Q_{30}$ as generating coordinates. At the mean-field level only nuclei with neutron numbers $130 \leq N \leq 138$ exhibit octupole deformed HFB ground states. However, for all the studied nuclei, the MFPESs and PPPESs are rather soft along the $Q_{30}$-direction. As a result, once correlations associated with parity restoration and quadrupoleoctupole configuration mixing are included simultane- 

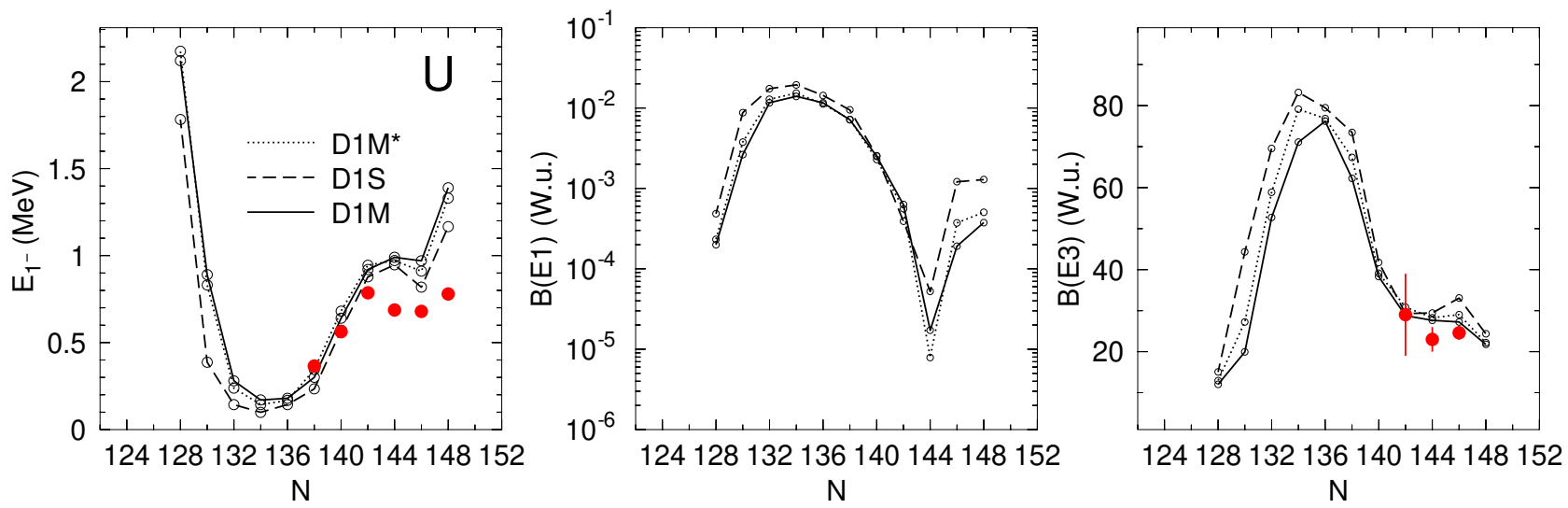

FIG. 11: (Color online) Same as Fig 10 but for different parametrizations of the Gogny force (D1M full line, D1M* dotted line and D1S dashed line).

ously within the 2D-GCM approach, their ground states turn out to be (dynamically) octupole deformed, albeit with the largest octupole deformation effects still corresponding to $N=132-138$ isotopes. Moreover, within the 2D-GCM approach, the correlation energies display a weaker dependence on the neutron number. Given the range of variation of those 2D-GCM correlation energies, they should be included in the fitting protocol of improved versions of the Gogny-EDF. Using the correlated 2D-GCM states, we have studied the systematic of the $1^{-}$energy splittings as well as $B(E 1)$ and $B(E 3)$ reduced transition probabilities in the considered isotopic chains. The predicted values compare reasonably well with the available experimental data. They point towards a (dynamically) enhanced octupolarity for $N=132-138$ isotopes while octupole-vibrational states have been found for other nuclei. The comparison with 1D-GCM results 53 reveals that, for the studied nuclei, the quadrupoleoctupole coupling is weak and to a large extent the properties of negative parity states (i.e., energy splittings and reduced transition probabilities) can be reasonably well described in terms of the octupole degree of freedom alone.

\section{Acknowledgments}

The work of LMR was supported by Spanish Ministry of Economy and Competitiveness (MINECO) Grants No. PGC2018-094583-B-I00.
[1] I. Ahmad and P. A. Butler, Ann. Rev. Nucl. Part. Sci. 43, 71 (1993).

[2] P. A. Butler, J. Phys. G 43, 073002 (2016).

[3] P. A. Butler and L. Willmann, Nucl. Phys. News 25, 12 (2015).

[4] P.A. Butler and W. Nazarewicz, Rev. Mod. Phys. 68, 349 (1996).

[5] S. K. Tandel, M. Hemalatha, A. Y. Deo, S. B. Patel, R. Palit, T. Trivedi, J. Sethi, S. Saha, D. C. Biswas and S. Mukhopadhyay, Phys. Rev. C 87, 034319 (2013).

[6] L. P. Gaffney et al., Nature 497, 199 (2013).

[7] H. J. Li, S. J. Zhu, J. H. Hamilton, E. H. Wang, A. V. Ramayya, Y. J. Chen, J. K. Hwang, J. Ranger, S. H. Liu, Z. G. Xiao, Y. Huang, Z. Zhang, Y. X. Luo, J. O. Rasmussen, I. Y. Lee, G. M. Ter-Akopian, Y. T. Oganessian and W. C. Ma, Phys. Rev. C 90, 047303 (2014).

[8] I. Ahmad, R. R. Chasman, J. P. Green, F. G. Kondev and S. Zhu, Phys. Rev. C 92, 024313 (2015).

[9] B. Bucher et al., Phys. Rev. Lett. 116, 112503 (2016).

[10] B. Bucher et al, Phys. Rev. Lett. 118, 152504 (2017).

[11] P. A. Butler, L. P. Gaffney, et al, Phys. Rev. Lett. 124,
042503 (2020).

[12] M. M. R. Chishti, D. ODonnell, et al Nature Physics (2020)

[13] P. Möller and J.R. Nix, Nucl. Phys. A361, 117 (1981).

[14] G.A. Leander, R.K. Sheline, P. Möller, P. Olanders, I. Ragnarsson, and A.J. Sierk, Nucl. Phys. A388, 452 (1982).

[15] W. Nazarewicz et al., Nucl. Phys. A429, 269 (1984).

[16] W. Nazarewicz and S.L. Tabor, Phys. Rev. C 45, 2226 (1992).

[17] M. Babilon, N.V. Zamfir, D. Kusnezov, E.A. McCutchan, and A. Zilges, Phys. Rev. C 72, 064302 (2005).

[18] N. Minkov, P. Yotov, S. Drenska, W. Scheid, D. Bonatsos, D. Lenis, and D. Petrellis, Phys. Rev. C 73, 044315 (2006).

[19] K. Nomura, D. Vretenar, T. Niksic and Bing-Nan Lu, , Phys. Rev. C 89, 024312 (2014).

[20] K. Nomura, T. Niksic and D. Vretenar, Phys. Rev. C 97, 024317 (2018).

[21] K. Nomura, D. Vretenar and B. -N. Lu, Phys. Rev. C 88, 021303 (2013).

[22] K. Nomura, R. Rodríguez-Guzmán and L. M. Robledo, 
Phys. Rev. C 92, 014312 (2015).

[23] S. Marcos, H. Flocard, and P.H. Heenen, Nucl. Phys. A410, 125 (1983).

[24] P. Bonche, P. -H. Heenen, H. Flocard, and D. Vautherin, Phys. Lett. B175, 387 (1986).

[25] P. Bonche, J.S. Krieger, M.S. Weiss, J. Dobaczewski, H. Flocard, and P.-H. Heenen, Phys. Rev. Lett. 66, 876 (1991).

[26] P.-H. Heenen, J. Skalski, P.Bonche, and H. Flocard, Phys. Rev. C 50,802 (1994).

[27] J. Erler, K. Langanke, H. P. Loens, G. Martínez-Pinedo and P.-G. Reinhard, Phys. Rev. C 85, 025802 (2012).

[28] S. Ebata and T. Nakatsukasa, Physica Scr. 92, 064005 (2017).

[29] L.M. Robledo, J.L. Egido, J.F. Berger, and M. Girod, Phys. Lett. B187, 223 (1987).

[30] L.M. Robledo, J.L. Egido, B. Nerlo-Pomorska, and K. Pomorski, Phys. Lett. B201, 409 (1988).

[31] J.L. Egido and L.M. Robledo, Nucl. Phys. A518, 475 (1990).

[32] J.L. Egido and L.M. Robledo, Nucl. Phys. A524, 65 (1991).

[33] E. Garrote, J.L. Egido, and L.M. Robledo, Phys. Rev. Lett. 80, 4398 (1998); Nucl. Phys. A654, 723c (1999).

[34] L.M. Robledo, M. Baldo, P. Schuck, and X. Viñas, Phys. Rev. C 81,034315 (2010).

[35] J.L. Egido and L.M. Robledo, Nucl. Phys. A545, 589 (1992).

[36] R. Rodríguez-Guzmán, Y. M. Humadi and L. M. Robledo, Eur. Phys. J. A, 56, 43 (2020).

[37] W. H. Long, J. Meng, N. Van Giai and S. G. Zhou, Phys. Rev. C 69, 034319 (2004).

[38] S. Y. Xia, H. Tao, Y. Lu, Z. P. Li, T. Niksic and D. Vretenar, Phys. Rev. C 96, 054303 (2017).

[39] Z. Xu and Z.-P. Li, Chinese Phys. C 41, 124107 (2017).

[40] S. E. Agbemava, A. V. Afanasjev and P. Ring, Phys. Rev. C 93, 044304 (2016).

[41] S. E. Agbemava and A. V. Afanasjev, Phys. Rev. C 96, 024301 (2017).

[42] Y. Cao, S. E. Agbemava, A. V. Afanasjev, W. Nazarewicz and E. Olsen, arXiv:2004.01319 1 [nucl-th].

[43] Rémi N. Bernard, Luis M. Robledo and Tomás R. Rodríguez, Phys. Rev C 93, 061302 (2016).

[44] L. M. Robledo and R. Rodríguez-Guzmán, J. Phys. G: Nucl. Part. Phys. 39, 105103 (2012).

[45] J. F. Berger, M. Girod, and D. Gogny, Nucl. Phys. A 428, 23c (1984).

[46] F. Chappert, M. Girod, and S. Hilaire, Phys. Lett. B 668, 420 (2008).

[47] S. Goriely, S. Hilaire, M. Girod and S. Péru, Phys. Rev. Lett. 102, 242501 (2009).

[48] J. Dechargé and D. Gogny, Phys. Rev. C 21, 1568 (1980).
[49] M. Baldo, P. Schuck and X. Viñas, Phys. Lett. B 663, 390 (2008).

[50] L. M. Robledo, M. Baldo, P. Schuck and X. Viñas, Phys. Rev. C 77, 051301 (2008).

[51] L. M. Robledo, M. Baldo, P. Schuck and X. Viñas, Phys. Rev. C 81, 034315 (2010).

[52] M. Baldo, L. M. Robledo, P. Schuck and X. Viñas, J. Phys. G: Nucl. Part. Phys. 37, 064015 (20110).

[53] L. M. Robledo and J. F. Bertsch, Phys. Rev. C 84, 054302 (2011).

[54] L. M. Robledo, J. Phys. G: Nucl. Part. Phys. 42055109 (2015)

[55] R. Rodríguez-Guzmán, L. M. Robledo and P. Sarriguren, Phys. Rev. C 86, 034336 (2012).

[56] P. Ring and P. Schuck, The Nuclear Many-Body Problem (Springer, Berlin-Heidelberg-New York) (1980).

[57] L. M. Robledo and P. A. Butler, Phys. Rev C 88, 051302 (2013).

[58] L. M. Robledo, Tomás R. Rodríguez and R. RodríguezGuzmán, J. Phys. G: Nucl. Part. Phys. 46, 013001 (2019).

[59] R. Rodríguez-Guzmán, P. Sarriguren, L.M. Robledo and J.E. García-Ramos, Phys. Rev. C 81, 024310 (2010).

[60] R. Rodríguez-Guzmán, P. Sarriguren and L.M.Robledo, Phys. Rev. C 83, 044307 (2011).

[61] R. Rodríguez-Guzmán and L. M. Robledo, Phys. Rev. C 89, 054310 (2014).

[62] R. Rodríguez-Guzmán and L. M. Robledo, Eur. Phys. J. A 53, 245 (2017).

[63] T. Kibédi and R. Spear, Atomic Data and Nuclear Data Tables 80, 35 (2002).

[64] L.M. Robledo and G.F. Bertsch, Phys. Rev C 84, 014312 (2011).

[65] R. R. Rodríguez-Guzmán, J. L. Egido, and L.M. Robledo, Nucl. Phys. A709, 201 (2002).

[66] J.L Egido and L.M. Robledo, Lecture Notes in Physics 641, 269 (2004).

[67] L. M. Robledo, Int. J. of Mod. Phys. E 16, 337 (2007).

[68] L. M. Robledo, J. Phys. G: Nucl. Part. Phys. 37, 064020 (2010).

[69] K. Hara, A. Hayashi and P.Ring, Nucl. Phys. A385, 14 (1982).

[70] P. Bonche, J. Dobaczewski, H. Flocard, P.-H. Heenen and J. Meyer, Nucl. Phys. A510, 466 (1990).

[71] L. M. Robledo, Phys. Rev. C 46, 238 (1992).

[72] L. M. Robledo and J. F. Bertsch, Phys. Rev. C 86, 054306 (2012)

[73] L. M. Robledo, Eur. Phys. J. A 52, 300 (2016).

[74] C. Gonzalez-Boquera, M. Centelles, X. Viñas and L.M. Robledo, Phys. Lett. B779 (2018) 195. 